PROCEEDINGS OF THE

AMERICAN MATHEMATICAL SOCIETY

Volume 136, Number 10, October 2008, Pages 3601-3609

S 0002-9939(08)09344-1

Article electronically published on May 8, 2008

\title{
A COMPACTIFICATION OF THE MODULI SPACE OF POLYNOMIALS
}

\author{
MASAYO FUJIMURA AND MASAHIKO TANIGUCHI
}

(Communicated by Mei-Chi Shaw)

\begin{abstract}
In this paper, we introduce a compactification of the moduli space of polynomial maps with a fixed degree $n(\geq 2)$ such that the map from it to $\mathbb{P}^{n-1}(\mathbb{C})$ defined by using the elementary symmetric functions of multipliers at fixed points is a continuous surjection.
\end{abstract}

\section{INTRODUCTION AND MAIN THEOREMS}

Let $n \geq 2$ and Poly $_{n}$ be the set of all polynomial maps of $\mathbb{C}$ to itself with degree $n$. We say that two maps $p_{1}$ and $p_{2}$ in Poly ${ }_{n}$ are affine conjugate if there exists a biholomorphic automorphism $g$ of $\mathbb{C}$ such that $g \circ p_{1} \circ g^{-1}=p_{2}$. The moduli space of polynomial maps with degree $n$ is the set of all affine conjugacy classes of maps in Poly $_{n}$, and is denoted by MPoly ${ }_{n}$. Here, recall that a natural complex orbifold structure can be introduced on MPoly ${ }_{n}$.

Remark 1.1. These spaces were investigated by Branner and Hubbard [2, 3], and Milnor [12] in the case of degree 3, and then by the first author in general cases (cf. [9]).

Several kinds of compactification of MPoly ${ }_{n}$ have been considered. One is given as the closure in the GIT compactification of the moduli space of rational maps, which is defined in [14. (See also [4].) DeMarco and McMullen introduced one by using tree representations ([5]), and also DeMarco discussed another one in [4]. Here, we show that the extended moduli space introduced by the first author in [8] (also cf. 9], Introduction) gives a natural compactification of MPoly $_{n}$ with a suitable topology.

Definition 1.2 (Extended moduli spaces). The extended moduli space $\widehat{\mathrm{M}}_{n}$ of degree $n$ is the set of all collections $X=\left\{\left\langle p_{j}\right\rangle\right\}_{j=1}^{J}$ of the affine conjugacy classes $\left\langle p_{j}\right\rangle$ of polynomial maps $p_{j}$ with degree $n_{j}^{X}(\geq 2)$ such that $N_{X}=\sum_{j=1}^{J} n_{j}^{X} \leq n$. Here we include the empty collection in the extended moduli space.

By identifying the affine conjugacy class $\langle p\rangle$ of $p \in$ Poly $_{n}$ with the collection $\{\langle p\rangle\}$ consisting of this class only, we consider $\mathrm{MPoly}_{n}$ as a subset of $\widehat{\mathrm{M}}_{n}$.

Here, by definition, an element $X$ of $\widehat{\mathrm{M}}_{n}$ is an unordered set of affine conjugacy classes. But for the sake of convenience, we fix an order temporarily, and represent

Received by the editors June 25, 2007, and, in revised form, September 3, 2007.

2000 Mathematics Subject Classification. Primary 32G99; Secondary 37F10, 30C15.

The second author is partially supported by Grand-in-Aid for Scientific Research 19540181.

(C) 2008 American Mathematical Society Reverts to public domain 28 years from publication 
$X$ as $\left\{\left\langle p_{j}\right\rangle\right\}_{j=1}^{J}$. Then, we can associate $X$ with a collection of sets of numbers as follows. For $X=\left\{\left\langle p_{j}\right\rangle\right\}_{j=1}^{J}$, assume that the fixed points of $p_{j}$, counted including multiplicities, are represented by numbers

$$
n_{1}^{X}+\cdots+n_{j-1}^{X}+1, \cdots, n_{1}^{X}+\cdots+n_{j}^{X}
$$

for every $j$. We call the partition of $\{1, \cdots, n\}$ into

$$
\begin{aligned}
& E_{0}^{X}=\left\{N_{X}+1, \cdots, n\right\}, \\
& E_{1}^{X}=\left\{1, \cdots, n_{1}^{X}\right\}, \\
& \cdots, \\
& E_{J}^{X}=\left\{n_{1}^{X}+\cdots+n_{J-1}^{X}+1, \cdots, N_{X}\right\}
\end{aligned}
$$

the partition associated to $X=\left\{\left\langle p_{j}\right\rangle\right\}_{j=1}^{J}$. Here, if $X$ is the empty collection, then we set $J=0$ and $E_{0}^{X}=\{1, \cdots, n\}$.

Definition 1.3 (Natural topology on the extended moduli space). Fix a point $X=\left\{\left\langle p_{j}\right\rangle\right\}_{j=1}^{J}$ of $\widehat{\mathrm{M}}_{n}$, and let $n_{j}^{X}, N_{X}$, and $\left\{E_{0}^{X}, \cdots, E_{J}^{X}\right\}$ be as above.

For every $\epsilon>0$, let $U_{\epsilon}(X)$ be the set of all points in $\widehat{\mathrm{M}}_{n}$, i.e. of all collections $Y=\left\{\left\langle q_{h}\right\rangle\right\}_{h=1}^{H}$ of the affine conjugacy classes of polynomial maps $q_{h}$ with degree $n_{h}^{Y}$ such that $N_{Y}=\sum_{h=1}^{H} n_{h}^{Y} \leq n$, which satisfy the following conditions:

(1) (Marking) There is a family of mutually disjoint subsets $P_{1}, \cdots, P_{H}$ of $\{1, \cdots, J\}$ such that $\bigcup_{h=1}^{H} P_{h}=\{1, \cdots, J\}$ and

$$
n_{h}^{Y} \geq N_{h}^{X}=\sum_{j \in P_{h}} n_{j}^{X}
$$

for every $h=1, \cdots, H$ (which implies that $N_{Y} \geq N_{X}$ ). Here, some of $P_{h}$ may be empty and then we set $N_{h}^{X}=0$. (In particular, $H$ may be greater than $J$.)

Furthermore, if $N_{X}>0$, then there is an injection

$$
\iota:\left\{1, \cdots, N_{X}\right\} \rightarrow\left\{1, \cdots, N_{Y}\right\}
$$

such that the image of $\bigcup_{j \in P_{h}} E_{j}^{X}$ by $\iota$ is contained in $E_{h}^{Y}$ for every $h$, where $\left\{E_{0}^{Y}, \cdots, E_{H}^{Y}\right\}$ is the partition associated to $Y=\left\{\left\langle q_{h}\right\rangle\right\}_{h=1}^{H}$.

(2) (Convergence) For every $k$ with $1 \leq k \leq N_{X}$, denote by $p_{j(k)}$ and $q_{h(k)}$ the polynomial maps in $\left\{p_{j}\right\}_{j=1}^{J}$ and $\left\{q_{h}\right\}_{h=1}^{H}$ having fixed points represented by $k$ and $\iota(k)$, respectively. We normalize $p_{j(k)}$ and $q_{h(k)}$ so that these fixed points are 0 and all coefficients of $z^{r}$ with $r \neq 1$ have the absolute values not greater than 1 and at least one of them is 1 . Then, among polynomial maps obtained from $p_{j(k)}$ and $q_{h(k)}$ by this normalization, we can find polynomial maps $\tilde{p}_{k}$ and $\tilde{q}_{k}$, respectively, such that

$$
\left|\tilde{p}_{k}-\tilde{q}_{k}\right|<\epsilon
$$

on $\{z \in \mathbb{C}|| z \mid<1 / \epsilon\}$.

(3) (Degeneration) For every $l$ with $1 \leq l \leq N_{Y}$ not in the image of $\iota$, let $q_{h(l)}$ be the polynomial map in $\left\{q_{h}\right\}_{h=1}^{H}$ having a fixed point represented by $l$, and $m_{l}(Y)$ be the multiplier of $q_{h(l)}$ at the fixed point. Then we have

$$
\left|m_{l}(Y)\right|>\frac{1}{\epsilon} .
$$


Finally, take

$$
\left\{U_{\epsilon}(X) \mid \epsilon>0, X \in \widehat{\mathrm{M}}_{n}\right\}
$$

as basic open sets of $\widehat{\mathrm{M}}_{n}$, and we have a topology on $\widehat{\mathrm{M}}_{n}$.

Remark 1.4. If $J=0$, i.e. $X$ is the empty collection, then we assume that $N_{X}=0$ and $P_{h}=\emptyset$ for every $h$, and hence conditions (1) and (2) hold trivially.

Proposition 1.5. The set $\mathrm{MPoly}_{n}$ is open in $\widehat{\mathrm{M}}_{n}$.

Proof. Fix $X \in$ MPoly $_{n}$. Then $X$ consists of a single affine conjugacy class of a polynomial map $p$ of degree $n$. Fix $\epsilon>0$ arbitrarily. Then the condition (1) implies that every point of $U_{\epsilon}(X)$ again consists of a single affine conjugacy class of a polynomial $p$ of degree $n$. Thus $U_{\epsilon}(X) \subset$ MPoly $_{n}$.

Proposition 1.6. Points $Y_{m} \in$ MPoly $_{n}$ tend to the empty collection if and only if the multipliers $m_{h}\left(Y_{m}\right)$ tend to $\infty$ as $m$ tend to $\infty$ for every $h(=1, \cdots, n)$.

Proof. By definition, $Y_{m} \in$ MPoly $_{n}$ tend to the empty collection if and only if, for every $\epsilon>0$, there is an $m_{0}$ such that

$$
\left|m_{h}\left(Y_{m}\right)\right|>\frac{1}{\epsilon}
$$

for every $h$ and every $m \geq m_{0}$, which is clearly equivalent to the fact that the multipliers $m_{h}\left(Y_{m}\right)$ tend to $\infty$ as $m$ tend to $\infty$ for every $h$.

Corollary 1.7. The points $Y_{m}=\left\{\left\langle q_{m}\right\rangle\right\} \in \widehat{\mathrm{M}}_{n}$ with $q_{m}(z)=z^{n}+z-m^{n}$ tend to the empty collection as $m$ tends to $\infty$.

Now the main theorem of this paper is the following one.

Theorem 1.8. The extended moduli space $\widehat{\mathrm{M}}_{n}$ of degree $n$ equipped with the topology defined above is compact, and $\mathrm{MPoly}_{n}$ is dense in it.

The proof will be given in $\S 2$.

Next, we define a map

$$
\widehat{\Psi}_{n}: \widehat{\mathrm{M}}_{n} \rightarrow \mathbb{P}^{n-1}(\mathbb{C}) .
$$

Definition 1.9 (Extended multiplier representation). Let $X=\left\{\left\langle p_{j}\right\rangle\right\}_{j=1}^{J}$ be an element of $\widehat{\mathrm{M}}_{n}$, and let $n_{j}^{X}$ and $N_{X}$ be as before. Let $\sigma_{k}\left(k=1, \cdots, N_{X}\right)$ be the elementary symmetric functions of multipliers $\left\{m_{k}\right\}_{k=1}^{N_{X}}$ at finite fixed points of all $p_{j}$ counted including multiplicities:

$$
\begin{aligned}
& \sigma_{1}=m_{1}+m_{2}+\cdots+m_{N_{X}}, \\
& \cdots, \\
& \sigma_{r}=\sum_{j_{1}<j_{2}<\cdots<j_{r}} m_{j_{1}} m_{j_{2}} \cdots m_{j_{r}}, \\
& \cdots, \\
& \sigma_{N_{X}}=m_{1} m_{2} \cdots m_{N_{X}} .
\end{aligned}
$$

We define the map $\widehat{\Psi}_{n}$ by sending $X$ to the point of $\mathbb{P}^{n-1}(\mathbb{C})$ represented by

$$
(\underbrace{0: \cdots: 0}_{n-N_{X}}: 1: \sigma_{1}: \cdots: \sigma_{N_{X}-2}: \sigma_{N_{X}}) \text {. }
$$


Finally, we map the empty collection to the point represented by

$$
(\underbrace{0: \cdots: 0}_{n-1}: 1) \text {. }
$$

Here it is well-known that for every polynomial with degree $n$ the unique linear relation between the elementary symmetric functions $\left\{\sigma_{k}\right\}_{k=1}^{n}$ of multipliers at finite fixed points is

$$
n+\sum_{k=1}^{n}(-1)^{k}(n-k) \sigma_{k}=0
$$

which is called Fatou's index theorem. This relation still holds in the circumstance as in the above definition when we replace $n$ by $N_{X}$. This is the reason why we delete $\sigma_{N_{X}-1}$ in the definition of $\widehat{\Psi}_{n}$.

Now, we conclude the following theorem.

Theorem 1.10 (Cf. [8], Theorem 3.2). The map

$$
\widehat{\Psi}_{n}: \widehat{\mathrm{M}}_{n} \rightarrow \mathbb{P}^{n-1}(\mathbb{C})
$$

defined as above is a continuous surjection.

Here recall that $\widehat{\Psi}_{n}$ restricted to MPoly $_{n}$ can be considered as a map into $\mathbb{C}^{n-1}$, which is never surjective if $n \geq 4$ ([9], Theorem 2). Also we have the following corollary of [9], Theorem 6 .

Theorem 1.11. For a generic point $x$ of $\mathbb{P}^{n-1}(\mathbb{C})$, the preimage $\widehat{\Psi}_{n}^{-1}(x)$ consists of exactly $(n-2)$ ! points of $\widehat{\mathrm{M}}_{n}$.

The proofs of Theorems 1.10 and 1.11 will be given in $\S 3$.

Remark 1.12. 1) The construction of our compactification resembles that of the Deligne-Mumford one due to Bers for the moduli space of compact Riemann surfaces ([1] and cf. [11]). Also see [14, the Remark after Corollary 1.6.

2) There is another deformation space concerning Poly ${ }_{n}$. We say that two maps $p_{1}$ and $p_{2}$ in Poly $_{n}$ determine the same isomorphism class if there is a biholomorphic automorphism $g$ of $\mathbb{C}$ such that $p_{2}=p_{1} \circ g$. The (classical) Hurwitz space $H_{0, n}[n]$ of genus 0 and degree $n$ with type $(n)$ is the set consisting of all isomorphism classes of polynomial maps of degree $n$ in general position. Recall that a natural compactification of the Hurwitz space was introduced in [7] and [13]. (Also cf. [6].)

\section{Proof of Theorem 1.8}

First, we show that MPoly $_{n}$ is dense in $\widehat{\mathrm{M}}_{n}$. By Corollary 1.7, we start with a point $X=\left\{\left\langle p_{j}\right\rangle\right\}_{j=1}^{J}$ in $\widehat{\mathrm{M}}_{n}$ which is not the empty collection, i.e. with $J \geq 1$.

First, assume that $J=1$ but $(2 \leq) n_{1}=n_{1}^{X}<n$. Here, we may write $p_{1}$ as

$$
p_{1}(z)=z+\prod_{\mu=1}^{n_{1}}\left(z-a_{\mu}\right) \text {. }
$$

Set

$$
p_{1} \#^{r}(z)=z+\frac{r-z}{r} \prod_{\mu=1}^{n_{1}}\left(z-a_{\mu}\right) .
$$


Then $p_{1} \#^{r}$ are polynomials with degree $n_{1}+1$ converging to $p_{1}$ uniformly on compact sets in $\mathbb{C}$ as $r$ tend to $\infty$. Also, since $n_{1} \geq 2$, the multipliers at $r$ tend to $\infty$ as do $r$. Thus the following lemma is clear.

Lemma 2.1. $Y_{r}=\left\{\left\langle p_{1} \#^{r}\right\rangle\right\}$ converge to $X$ as $r$ tend to $\infty$.

If $J>1$, then we also consider $p_{2}$ and set $n_{2}=n_{2}^{X}(\geq 2)$. We may write $p_{2}$ as

$$
p_{2}(z)=z+\prod_{\nu=1}^{n_{2}}\left(-z+b_{\nu}\right) \text {. }
$$

We set

$$
p_{1} \#^{r} p_{2}(z)=z+\frac{1}{r^{\left(n_{2}-1\right) n_{2}}} \prod_{\mu=1}^{n_{1}}\left(z-a_{\mu}\right) \prod_{\nu=1}^{n_{2}}\left(-z+r^{n_{2}-1}+r^{n_{2}-n_{1}} b_{\nu}\right) .
$$

Then $p_{1} \#^{r} p_{2}$ are polynomials with degree $n_{1}+n_{2}$ converging to $p_{1}$ uniformly on compact sets in $\mathbb{C}$ as $r$ tend to $\infty$. Also, we have

Lemma 2.2. $Y_{r}=\left\{\left\langle p_{1} \#^{r} p_{2}\right\rangle,\left\langle p_{3}\right\rangle, \cdots,\left\langle p_{J}\right\rangle\right\}$ converge to $X$ as $r$ tend to $+\infty$.

Proof. The polynomials $p_{1} \#^{r} p_{2}(z)$ are conjugate to

$$
z+\frac{1}{r^{\left(n_{1}-1\right) n_{1}}} \prod_{\mu=1}^{n_{1}}\left(z+r^{n_{1}-1}-r^{n_{1}-n_{2}} a_{\mu}\right) \prod_{\nu=1}^{n_{2}}\left(-z+b_{\nu}\right)
$$

by the affine automorphisms

$$
V_{r}(z)=\frac{z-r^{n_{2}-1}}{r^{n_{2}-n_{1}}}
$$

which converge to $p_{2}$ uniformly on compact sets in $\mathbb{C}$ as $r$ tend to $\infty$. Thus the assertion follows.

Proof of denseness. Take a sufficiently large $r$, and we have an element $\left\{\left\langle p_{1} \#^{r}\right\rangle\right\}$ or $\left\{\left\langle p_{1} \#^{r} p_{2}\right\rangle,\left\langle p_{3}\right\rangle, \cdots,\left\langle p_{J}\right\rangle\right\}$ of $\widehat{\mathrm{M}}_{n}$ which is arbitrarily near to $X$ by Lemmas 2.1] and 2.2 and which satisfies that the degree of $p_{1} \#^{r}$ is $n_{1}+1$ or (the degree of $p_{1} \#^{r} p_{2}$ is $n_{1}+n_{2}$ but) the number of the conjugacy classes decreases from $J$ to $J-1$, respectively.

Repeating such an approximation by a finite number of times, we have an element of MPoly $_{n}$ arbitrarily near to $X$. Thus we conclude that MPoly ${ }_{n}$ is dense in $\widehat{M}_{n}$.

Example 2.3. The points $Y_{m}=\left\{\left\langle q_{m}\right\rangle\right\} \in \widehat{\mathrm{M}}_{n}(n \geq 3)$ with $q_{m}(z)=z^{3}-m z^{2}+z$ converge to $X=\{\langle q\rangle\}$ with $q(z)=z^{2}+z$ as $m$ tend to $\infty$.

Indeed, the fixed points of $q_{m}$ are 0 (double) and $m$, with multipliers 1 and $m^{2}+1$, respectively. Associate the numbers 1,2 to 0 and normalize $q_{m}$ as before with respect to 0 , and we have $\tilde{q}_{1}[m](z)=m^{-2} z^{3}+z^{2}+z$, which converge to $q(z)$ uniformly on compact sets in $\mathbb{C}$.

Example 2.4. The points $Y_{m}=\left\{\left\langle q_{m}\right\rangle\right\} \in \widehat{\mathrm{M}}_{n}(n \geq 4)$ with $q_{m}(z)=z^{4}-2 m z^{3}+$ $m^{2} z^{2}+z$ converge to $\{\langle q\rangle,\langle q\rangle\}$ with $q(z)=z^{2}+z$ as $m$ tend to $\infty$.

Indeed, the fixed points of $q_{m}$ are 0 (double) and $m$ (double) with multiplier 1. We associate the number 1,2 to 0 and 3,4 to $m$. Normalize $q_{m}$ as before with respect to 0 and $m$, and get

$\tilde{q}_{1}[m](z)=m^{-6} z^{4}-2 m^{-3} z^{3}+z^{2}+z$ and $\tilde{q}_{3}[m](z)=m^{-6} z^{4}+2 m^{-3} z^{3}+z^{2}+z$, respectively, both of which converge to $q(z)$ uniformly on compact sets in $\mathbb{C}$. 
Next, the space $\widehat{\mathrm{M}}_{n}$ equipped with the topology defined above satisfies the second countability axiom. (Actually, the topology has a countable base

$$
\left\{U_{\epsilon_{u}}\left(X_{v}\right) \mid u, v \in \mathbb{Z}\right\}
$$

where $\epsilon_{u}$ moves over all positive rational numbers and $X_{v}$ over all collections of the affine conjugacy classes of polynomial maps with rational coefficients.) Hence to finish the proof of Theorem 1.8, it is enough to show sequential compactness of $\widehat{\mathrm{M}}_{n}$.

Let $\left\{Y_{l}\right\}_{l=1}^{\infty}$ be an arbitrary sequence of distinct points in $\widehat{\mathrm{M}}_{n}$. Here taking a subsequence if necessary, we may assume that every $Y_{l}$ consists of the same number of affine conjugacy classes, say $\left\{\left\langle q_{h}[l]\right\rangle\right\}_{h=1}^{H}$, and that the degree of $q_{h}[l]$ is a constant $n_{h}$ for every $l$. Set $N=\sum_{h=1}^{H} n_{h}$. We may also assume that all fixed points of all $q_{h}[l]$ are numbered so that the fixed point $z_{k}[l]$ represented by $k$ is a fixed point of $q_{h(k)}[l]$ with $h(k)$ independent of $l$ for every $k(=1, \cdots, N)$.

Let $m_{k}[l]$ be the multipliers of $q_{h(k)}[l]$ at the fixed point $z_{k}[l]$. Here, again taking a subsequence and renumbering the fixed points if necessary, we may assume that $m_{k}[l]$ converge to some value $m_{k}$ in $\mathbb{P}^{1}(\mathbb{C})$ as $l$ tend to $\infty$ for every $k$, and that $m_{k}=\infty$ if and only if $k$ satisfies $N^{\prime}<k \leq N$ with some $N^{\prime} \leq N$. Here, if $N^{\prime}=0$, then as in the proof of Proposition [1.6, $Y_{l}$ converge to the empty collection as $l$ tend to $\infty$.

If $N^{\prime}>0$, then $m_{1}[l]$ converge to a finite $m_{1}$ as $l$ tend to $\infty$. Let $\tilde{q}_{1}[l]$ be a polynomial map obtained from $q_{h(1)}[l]$ by the normalization as before with respect to $z_{1}[l]$ for every $l$. Then taking a subsequence if necessary, we may assume that $\tilde{q}_{1}[l]$ converge to a polynomial $\tilde{p}_{1}$ uniformly on compact sets in $\mathbb{C}$ as $l$ tend to $\infty$. Here from the definition, the degree of $\tilde{p}_{1}$ is not less than 2 , and the multiplier at the fixed point 0 is $m_{1}$.

Let $k$ satisfy that $1<k \leq N^{\prime}, q_{h(k)}[l]=q_{h(1)}[l]$ and the fixed points $\tilde{z}_{k}[l]$ of $\tilde{q}_{1}[l]$ corresponding to $z_{k}[l]$ are bounded. Then, we may further assume that $\tilde{z}_{k}[l]$ converge to a fixed point $\tilde{z}_{k}$ of $\tilde{p}_{1}$, which is represented also by $k$, as $l$ tend to $\infty$. Let Fix $_{1}$ be the set of all numbers representing fixed points of $\tilde{p}_{1}$ including multiplicities, and we have the following lemma.

Lemma 2.5. For every $k \in \mathrm{Fix}_{1}$, we normalize $q_{h(1)}[l]$ and $\tilde{p}_{1}$ as before with respect to $z_{k}[l]$ and $\tilde{z}_{k}$, respectively. Then among polynomial maps obtained by the normalization, we can find polynomial maps $\tilde{q}_{k}[l]$ and $\tilde{p}_{k}$, respectively, such that $\tilde{q}_{k}[l]$ converge to $\tilde{p}_{k}$ locally uniformly on $\mathbb{C}$ as $l$ tend to $\infty$.

Proof. If $k>1$ and $k \in \mathrm{Fix}_{1}$, take the conjugates of $\tilde{q}_{1}[l]$ by the affine automorphisms $U_{l}(z)=z-\tilde{z}_{k}[l]$. Then we have polynomials with bounded coefficients which converge to the conjugate of $\tilde{p}_{1}$ by $U(z)=z-\tilde{z}_{k}$, which implies the assertion.

Now let $k_{2}(>1)$ be the least number not contained in $\mathrm{Fix}_{1}$. Then starting from $q_{h\left(k_{2}\right)}[l]$, we also have a polynomial map $\tilde{p}_{k_{2}}$ satisfying the same assertion as in Lemma 2.5 with $k_{2}$ instead of 1.

Proof of compactness. Repeating the above arguments a finite number of times, we finally get a set of polynomial maps $\left\{\tilde{p}_{k}\right\}_{k=1}^{N^{\prime}}$. Let

$$
\left\{\operatorname{Fix}_{1}, \operatorname{Fix}_{k_{2}}, \cdots, \operatorname{Fix}_{k_{K}}\right\}
$$

be the corresponding partition of $\left\{1, \cdots, N^{\prime}\right\}$, and set

$$
X=\left\{\left\langle\tilde{p}_{1}\right\rangle,\left\langle\tilde{p}_{k_{2}}\right\rangle, \cdots,\left\langle\tilde{p}_{k_{K}}\right\rangle\right\} .
$$


Then from the construction, $Y_{l}$ converge to $X$ in $\widehat{\mathrm{M}}_{n}$ as $l$ tend to $\infty$.

\section{Proofs of Theorems 1.10 and 1.11}

First we show the following lemma.

Lemma 3.1. $\widehat{\Psi}_{n}$ is continuous.

Proof. If the multipliers $m_{k}(X)$ at the fixed point represented by $k$ are bounded for all $k$, then by condition $(2), m_{k}(X)$ are continuous. Since we define $\widehat{\Psi}_{n}$ by using the elementary symmetric functions of these multipliers, the assertion follows.

The case that some $m_{k}(X)$ are $\infty$ or tend to $\infty$ is similar. For the sake of simplicity, we give a proof only for the case that $m_{1}(X)$ are finite but tend to $\infty$, while the others are bounded, as $X$ tend to $X_{\infty}$. In this case, $\widehat{\Psi}_{n}(X)$ can also be represented as

$$
\left(\frac{1}{m_{1}(X)}: 1+\frac{\sigma_{1}^{\prime}(X)}{m_{1}(X)}: \cdots: \sigma_{n}^{\prime}(X)\right)
$$

with $\sigma_{1}^{\prime}(X)=\sum_{k=2}^{n} m_{k}(X), \cdots, \sigma_{n}^{\prime}(X)=\prod_{k=2}^{n} m_{k}(X)$, which converge to $\widehat{\Psi}_{n}\left(X_{\infty}\right)$ from the definition.

Proof of Theorem 1.10. Since $\widehat{\Psi}_{n}$ is continuous by Lemma 3.1 and $\widehat{\mathrm{M}}_{n}$ is compact by Theorem 1.8 , the image $\widehat{\Psi}_{n}\left(\widehat{\mathrm{M}}_{n}\right)$ is also compact in $\mathbb{P}^{n-1}(\mathbb{C})$. Hence to prove Theorem 1.10 it remains only to show that the image $\widehat{\Psi}_{n}\left(\widehat{\mathrm{M}}_{n}\right)$ is dense. But by Lemma 3.2 below, $\widehat{\Psi}_{n}\left(\right.$ MPoly $\left._{n}\right)$ is already dense in $\mathbb{P}^{n-1}(\mathbb{C})$.

Lemma 3.2. $\widehat{\Psi}_{n}\left(\right.$ MPoly $\left._{n}\right)$ is dense in $\mathbb{P}^{n-1}(\mathbb{C})$.

Proof. Let $\Omega$ be the set of all points $\left(s_{1}, \cdots, s_{n-2}, s_{n}\right) \in \mathbb{C}^{n-1}$ such that there is a solution $\left\{m_{1}, \cdots, m_{n}\right\}$ of the equation system

$$
\begin{aligned}
& s_{1}=m_{1}+m_{2}+\cdots+m_{n}, \\
& \cdots, \\
& s_{r}=\sum_{j_{1}<j_{2}<\cdots<j_{r}} m_{j_{1}} m_{j_{2}} \cdots m_{j_{r}}, \\
& \cdots, \\
& s_{n}=m_{1} m_{2} \cdots m_{n},
\end{aligned}
$$

with $m_{j} \neq 1$ for every $j$ and $\sum_{j \in S} \frac{1}{1-m_{j}} \neq 0$ for every proper subset $S$ of $\{1, \cdots, n\}$. Here, the unique linear relation between the elementary symmetric functions corresponds to $\sum_{j=1}^{n} \frac{1}{1-m_{j}}=0$. This equation between $\left\{m_{j}\right\}_{j=1}^{n}$ is independent of the equations $m_{j}=1$ and $\sum_{j \in S} \frac{1}{1-m_{j}}=0$ for every proper subset $S$ of $\{1, \cdots, n\}$. Since the map sending $\left(m_{1}, \cdots, m_{n}\right)$ to $\left(s_{1}, \cdots, s_{n}\right)$ is a finite-sheeted holomorphic branched covering projection of $\mathbb{C}^{n}$ onto itself, we see that $\Omega$ is a generic set in $\mathbb{C}^{n-1}$

On the other hand, 9], Theorem 4, implies that $\widehat{\Psi}_{n}\left(\mathrm{MPoly}_{n}\right)$ contains $\Omega$, which shows the assertion.

Proof of Theorem 1.11. Every element of $\widehat{\mathrm{M}}_{n}-\mathrm{MPoly}_{n}$ consists of either a single affine conjugacy class of a polynomial map with degree less than $n$ or of more than one class. Hence the set $\widehat{\mathrm{M}}_{n}-\mathrm{MPoly}_{n}$ is, at most, complex $n-2$ dimensional, which implies that $\mathbb{C}^{n-1}-\widehat{\Psi}_{n}\left(\widehat{\mathrm{M}}_{n}-\right.$ MPoly $\left._{n}\right)$ is a generic set in $\mathbb{P}^{n-1}(\mathbb{C})$. Thus it is enough to show that for a generic point in $\mathbb{C}^{n-1}$ the preimage of the point by 
$\widehat{\Psi}_{n}$ contains exactly $(n-2)$ ! points counted including multiplicities. But this is 9 , Theorem 6 .

As closing remarks, we discuss more details in the case that $n=2,3$, and 4 .

First, $\widehat{\mathrm{M}}_{2}-\mathrm{MPoly}_{2}$ consists of the empty collection only. Thus $\widehat{\mathrm{M}}_{2}$ is the onepoint compactification of $\mathrm{MPoly}_{2}$, and $\widehat{\mathrm{M}}_{2}$ is homeomorphic to $\mathbb{P}^{1}(\mathbb{C})$.

Next, $\widehat{\mathrm{M}}_{3}-$ MPoly $_{3}$ consists of the empty collection and a set which can be identified with $\mathrm{MPoly}_{2}$. Hence $\widehat{\mathrm{M}}_{3}$ is homeomorphic to $\mathbb{P}^{2}(\mathbb{C})$ and $\widehat{\Psi}_{3}$ gives a homeomorphism. Compare with [5], Theorem 11.1.

Now, $\widehat{M}_{4}-$ MPoly $_{4}$ consists of the empty collection and sets

$$
E_{2}, E_{3}, E_{2,2}
$$

which can be identified with $\mathrm{MPoly}_{2}, \mathrm{MPoly}_{3}$, and the symmetric product of $\mathrm{MPoly}_{2}$ with itself, respectively. Also we can describe the map $\widehat{\Psi}_{4}$ completely as follows (cf. [10, Theorem 3):

(1) If $x \in \mathbb{P}^{3}(\mathbb{C})$ is represented by $(1: 4: 6: 1)$, then $\widehat{\Psi}_{4}^{-1}(x)$ consists of infinite number of points.

(2) If $x$ is represented by either

(a) $\left(0: s_{1}: s_{2}: s_{4}\right)$ or

(b) $\left(1: s_{1}: s_{2}: s_{4}\right)(\neq(1: 4: 6: 1))$ satisfying the equation

$$
\begin{aligned}
& 54 s_{1}^{5}+\left(-81 s_{2}-27 s_{4}-135\right) s_{1}^{4}+\left(36 s_{2}^{2}-144 s_{2}-1008\right) s_{1}^{3} \\
& +\left(-4 s_{2}^{3}+360 s_{2}^{2}+\left(144 s_{4}+2976\right) s_{2}+576 s_{4}+4192\right) s_{1}^{2} \\
& +\left(-160 s_{2}^{3}-2176 s_{2}^{2}+\left(-384 s_{4}-6400\right) s_{2}-1280 s_{4}-5376\right) s_{1} \\
& +16 s_{2}^{4}+448 s_{2}^{3}+\left(-128 s_{4}+2176\right) s_{2}^{2}+\left(256 s_{4}+3840\right) s_{2} \\
& +256 s_{4}^{2}+768 s_{4}+2304=0,
\end{aligned}
$$

then $\widehat{\Psi}_{4}^{-1}(x)$ consists of a single point.

(3) Otherwise $\widehat{\Psi}_{4}^{-1}(x)$ consists of two points.

\section{ACKNOWLEDGEMENT}

The authors express their hearty thanks to the referee for valuable comments and helpful suggestions.

\section{REFERENCES}

1. L. Bers, On the space of Riemann surfaces with nodes, Bull. AMS. 80 (1974), 1219-1222. MR0361165 (50:13611)

2. B. Branner and J. H. Hubbard, The iteration of cubic polynomials, Part I: The global topology of parameter space, Acta Math. 160 (1988), 143-206. MR945011 (90d:30073)

3. The iteration of cubic polynomials, Part II: Patterns and parapatterns, Acta Math. 169 (1992), 229-325. MR1194004 (94d:30044)

4. L. DeMarco, Finiteness for degenerate polynomials, arXiv: math/0608800v1.

5. L. DeMarco and C. McMullen, Trees and the dynamics of polynomials, arXiv: math/0608759v1.

6. S. Diaz, On the Natanzon-Turaev compactification of the Hurwitz space, Proc. AMS 130 (2001), 613-618. MR1866008 (2002h:14042)

7. S. Diaz and D. Edidin, Towards the homology of Hurwitz spaces, J. Diff, Geom. 43 (1996), 66-98. MR 1424420 (98e:14028)

8. M. Fujimura, Projective moduli space for the polynomials, Dynam. Conti. Discrete Impul. Systems 13 (2006), 787-801. MR.2273342 (2007h:37061)

9. Comp. Meth. Funct. Th. 7 (2007), 345-360. 
10. M. Fujimura and K. Nishizawa, Some dynamical loci of quartic polynomials, J. Japan Soc. Symb. Alg. Compt. 11 (2005), 57-68.

11. Y. Imayoshi and M. Taniguchi, An introduction to Teichmüller spaces, Springer (Tokyo), 1992. MR.1215481 (94b:32031)

12. J. Milnor, Remarks on iterated cubic maps, Experiment. Math. 1 (1992), 5-24. MR 1181083 (94c:58096)

13. S. M. Natanzon and V. Turaev, A compactification of the Hurwitz spaces, Topology 38 (1999), 889-914. MR1679803 (2000b:57004)

14. J. Silverman, The space of rational maps on $\mathbb{P}^{1}$, Duke Math. J. 94 (1998), 41-77. MR.1635900 $(2000 \mathrm{~m}: 14010)$

Department of Mathematics, National Defense Academy, Yokosuka 239-8686, Japan

E-mail address: masayo@nda.ac.jp

Department of Mathematics, Nara Women's University, Nara 630-8506, Japan

E-mail address: tanig@cc.nara-wu.ac.jp 